\title{
First Practical Synthesis of Formamidine Ureas and Derivatives
}

\author{
Amy S. Ripka,* David D. Díaz, K. Barry Sharpless, and M.G. Finn* \\ Department of Chemistry and The Skaggs Institute for Chemical Biology, The Scripps Research \\ Institute, 10550 N. Torrey Pines Rd., La Jolla, CA 92037
}

\section{Supporting Information}

\section{EXPANDED DISCUSSION}

\section{Literature precedents}

Four formamidine urea structures (bearing $\mathrm{H}$-atom substitution at the central amidine carbon atom) are reported in the Beilstein database, as shown in Scheme S1. The only preparative details available (from the paper by Jentzsch \& Seefelder) describe the elaboration of formamidines (RNHCOH) with oxalyl, phosphoryl, or sulfuryl chloride, to imidoyl halide (chloroiminium) species with subsequent capture by amines.

\section{Scheme S1}
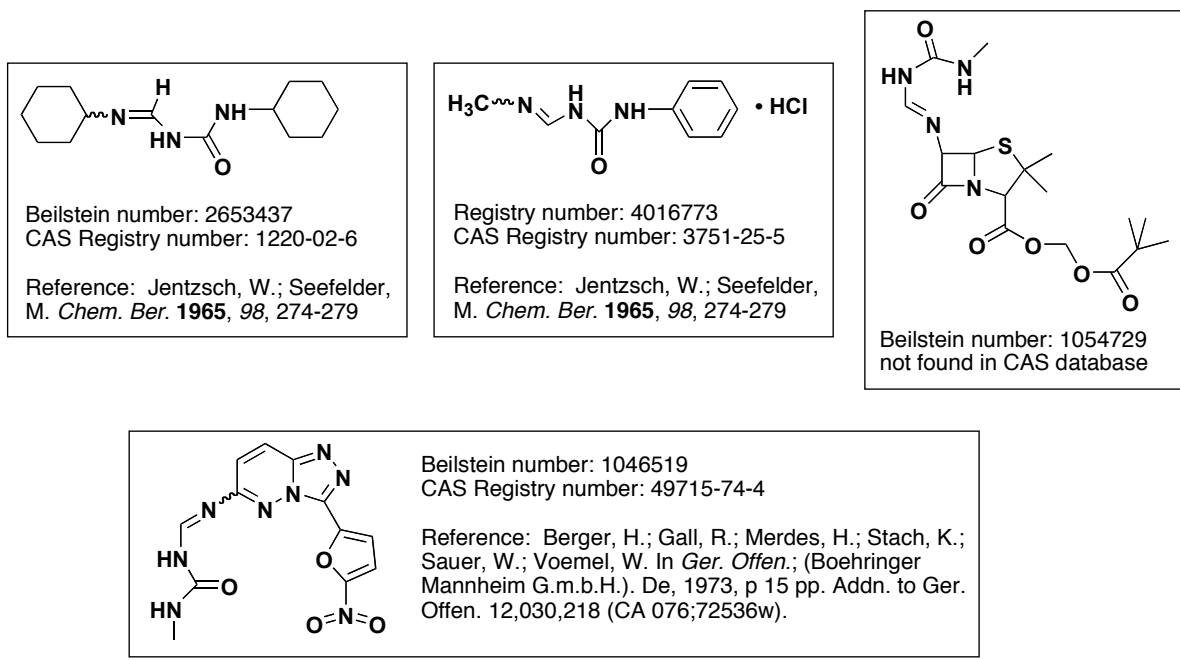

The vast majority of other amidine urea structures known have alkyl or aryl substitution at the central amidine carbon. The chemistry used to construct them is dominated by Vilsmeiertype sequences using $\mathrm{COCl}_{2}$ or $\mathrm{SOCl}_{2}$. Early examples include: Schwenker; Kolb. Tetrahedron 1969, 25, 5549-5551; Jentzsch, W.; Seefelder, M. Chem. Ber. 1973, 106, 105-114; Lecher, Gubernator J. Am. Chem. Soc. 1953, 75, 1087-1091 (activation using $\mathrm{Hg}^{\mathrm{II}}$ ). Thus, the process that we describe here is new and quite a bit more convenient than previous methods.

The more general amidine nucleus $\left[\mathrm{R}^{1} \mathrm{HN}-\mathrm{C}\left(\mathrm{R}^{2}\right)=\mathrm{NR} \mathrm{R}^{3}\right.$, with $\mathrm{R}^{2}$ usually $\left.\mathrm{H}\right]$ is available from isonitriles and amines under a variety of conditions. Prominent among these is mediation by electrophilic "soft" metals such as $\mathrm{Cu}, \mathrm{Hg}$, and $\mathrm{Ag},{ }^{1-10}$ and more recently In. ${ }^{11}$ This general scheme has also been applied to hydrazide and sulfonamide nucleophiles. ${ }^{12,13}$ A very early report describes the simple addition of amines to isonitriles in the presence of carboxylic acids. ${ }^{14}$ 
Other entries to amidines from isonitriles include the following.

- Pd-catalyzed assembly of isonitriles, aryl halides, and tin alkoxides, followed by amine displacement to give $\mathrm{C}$-aryl amidines. ${ }^{15}$

- Electrophilic activation by $N$-halobenzotriazoles (via intermediate imidoyl benzotriazoles). ${ }^{16}$

- Uncatalyzed attack by secondary amines on electron-deficient isonitriles, such as $\square$-cyano-

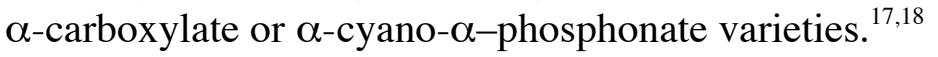

- Oxidative activation by (and incorporation of) chloramines $\mathrm{T},{ }^{19,20}$ sulfenyl chlorides, ${ }^{21}$ or elemental selenium. ${ }^{22}$

\section{Reaction design}

Our initial target, shown in Scheme S2, was a convenient synthesis of the imidazolone ring as a scaffold for displaying the urea functionality. Following the precedents of $\mathrm{Ugi}^{23}$ and Livinghouse, ${ }^{24-27}$ reaction of benzyl isocyanide with acetyl chloride was expected to form acetyl imidoylchloride $\mathbf{4}$, which would be captured by urea to give the acetyl imidourea $\mathbf{5}$. We hoped that $\mathbf{5}$ would be sufficiently stable to be treatable with reducing agent to provide the desired imidazalone 9. Of course, the observed course of the reaction suggests that $\mathbf{5}$ is sufficiently electrophilic to be attacked by another equivalent of urea. Furthermore, the rate of reaction of $\mathbf{5}$ with urea must be substantially faster than the reaction of $\mathbf{4}$, since the use of one equivalent of urea gives formamidine $\mathbf{1}$ rather than a buildup of intermediate $\mathbf{5}$.

Scheme S2<smiles>C#[N+]Cc1ccccc1</smiles><smiles>Cc1c(NCc2ccccc2)n(C)c(=O)n1C</smiles>

\section{Preliminary Survey of Reaction Conditions}

A preliminary survey of reaction conditions was undertaken in order to establish the dependence of the process on solvent, reactant stoichiometries, and acyl halide. The results are shown in Table S1 and were summarized in the text, but are repeated here for convenience. Yields were found to be highest in THF, and the use of three equivalents of urea is significantly superior to one equivalent. No additional benefit was provided by the use of greater amonts of urea (data not shown). Reaction yields were similar among the five acid chlorides used; note that none of them are particularly hindered. In each case, the corresponding $N$-acylurea 6 was 
detected as the major byproduct in solution, in $>60 \%$ yield with respect to acyl chloride used $\left(\mathrm{R}^{2}\right.$ $=\mathrm{Ph}$ is known $\left.^{28}\right)$. The use of acetyl chloride in catalytic amounts ( 0.1 equiv.) in $\mathrm{CH}_{3} \mathrm{CN}$ or THF gave no precipitated product.

Table S1. Isolated yields of $\mathbf{1 a}$ or $\mathbf{1 b}$ under various conditions. In all cases, 1.1 equivalents of acyl chloride was used relative to isonitrile; except for the results shown in the last column, all reactions were performed with 1.0 equivalent of 1,3-dimethylurea.

\begin{tabular}{|c|c|c|c|c|c|c|c|}
\hline \multirow{2}{*}{$\mathrm{R}^{1}$} & \multirow{2}{*}{$\mathrm{R}^{2}$} & \multicolumn{5}{|c|}{ solvent } & \multirow{2}{*}{$\begin{array}{c}3 \text { equiv. urea } \\
\text { THF }\end{array}$} \\
\hline & & $\mathrm{MeCN}$ & THF & $\mathrm{Et}_{2} \mathrm{O}$ & toluene & DMF & \\
\hline$t-\mathrm{Bu}$ & $\mathrm{CH}_{3}$ & $43 \%$ & $45 \%$ & & & & $73 \%$ \\
\hline$t-\mathrm{Bu}$ & cyclopropyl & $27 \%$ & $50 \%$ & & & & $74 \%$ \\
\hline$t-\mathrm{Bu}$ & isobutyl & $21 \%$ & $47 \%$ & & & & $62 \%$ \\
\hline$t-\mathrm{Bu}$ & $\mathrm{Ph}$ & $35 \%$ & $46 \%$ & & & & $57 \%$ \\
\hline$t-\mathrm{Bu}$ & $\mathrm{CH}_{2} \mathrm{Ph}$ & & & & & & $56 \%$ \\
\hline$t-\mathrm{Bu}$ & $n$-pentyl & & & & & & $63 \%$ \\
\hline $\mathrm{CH}_{2} \mathrm{Ph}$ & $\mathrm{CH}_{3}$ & $46 \%$ & $47 \%$ & $23 \%$ & $42 \%$ & $0 \%$ & $73 \%$ \\
\hline $\mathrm{CH}_{2} \mathrm{Ph}$ & cyclopropyl & $41 \%$ & $49 \%$ & $22 \%$ & $40 \%$ & $0 \%$ & $63 \%$ \\
\hline $\mathrm{CH}_{2} \mathrm{Ph}$ & isobutyl & $46 \%$ & $49 \%$ & $25 \%$ & $44 \%$ & & $63 \%$ \\
\hline $\mathrm{CH}_{2} \mathrm{Ph}$ & $\mathrm{Ph}$ & $12 \%$ & $50 \%$ & $21 \%$ & $33 \%$ & & $79 \%$ \\
\hline $\mathrm{CH}_{2} \mathrm{Ph}$ & $\mathrm{CH}_{2} \mathrm{Ph}$ & & & & & & $62 \%$ \\
\hline $\mathrm{CH}_{2} \mathrm{Ph}$ & $n$-pentyl & & & & & & $60 \%$ \\
\hline
\end{tabular}

\section{Survey of Electrophiles}

Among the most intriguing of the mechanistic questions posed by these results is the role of the acyl chloride. To probe this, the electrophilic scope of the process was explored as shown in Table S2. Yields are constant for a variety of aliphatic (entries 1-7), simple aromatic (entries 9-12), and vinylic (entries 15-18) acyl chlorides. Pivaloyl chloride fails (entry 8), presumably for steric reasons, and 2,6-dimethoxybenzoyl chloride is similarly poor (entry 13). Both steric and electronic factors may play a role in the latter case, since the hindered but electron deficient tetrachloroterephthaloyl dichloride gives the desired product (entry 14). Ineffective are oxalyl and sulfuryl chloride (entries 19-20), sulfonyl chlorides (entries 21-23), an acyl fluoride (entry 24), several activated alkyl chloride and bromide electrophiles (entries 25-27), a silyl chloride (entry 28), and protic acids (entry 29). Acyl bromides give lower yields (and require longer reaction times) than acyl chlorides (entries 30-31). Most interestingly, triphenylmethyl (trityl) chloride promotes the reaction in modest yield (entries 32-33); trityl bromide is less effective (entry 34). Note that little change in yield with trityl chloride is observed when only one equivalent of urea is employed (31\%) instead of the normal three equivalents (37\%), in contrast to the behavior of the reaction mediated by acid chlorides (Table S1). The electron-rich compound crystal violet shows no activity (entry 35 ). 
Table S2. Isolated yields of 1a from the following reaction conditions: 1.0 equiv. benzyl isocyanide, 3.0 equiv.; 1,3-dimethylurea; 1.1 equiv. of the indicated electrophile; $0.8 \mathrm{M}$ total solute in the indicated solvent; $9 \mathrm{~h}$ at room temperature.

\begin{tabular}{|c|c|c|c|c|c|c|c|}
\hline Entry & Electrophile & Solvent & Yield & Entry & Electrophile & Solvent & Yield \\
\hline$\overline{1}$ & $\overline{\mathrm{MeCOCl}}$ & $\overline{\text { THF }}$ & $\overline{73}$ & $\overline{19}$ & $(\mathrm{COCl})_{2}$ & $\mathrm{MeCN}$ & $\overline{0}$ \\
\hline 2 & $\triangleright-\mathrm{COCl}$ & THF & 75 & 20 & $\mathrm{SOCl} 2$ & $\mathrm{MeCN}$ & 0 \\
\hline 3 & $i$-PrCOCl & THF & 74 & 21 & & $\mathrm{MeCN}$ & 0 \\
\hline 4 & $n-\mathrm{C}_{5} \mathrm{H}_{11} \mathrm{COCl}$ & THF & 72 & 22 & & THF & 0 \\
\hline 5 & $\mathrm{PhCH}_{2} \mathrm{COCl}$ & THF & 71 & 23 & & $\mathrm{MeCN}$ & 0 \\
\hline 6 & $\mathrm{ClCH}_{2} \mathrm{COCl}$ & THF & 69 & 24 & $\mathrm{PhCOF}$ & $\mathrm{MeCN}$ & 0 \\
\hline 7 & $t-\mathrm{BuCH}_{2} \mathrm{COCl}$ & $\mathrm{MeCN}$ & 60 & 25 & & $\mathrm{MeCN}$ & 0 \\
\hline 8 & $t$-BuCOCl & $\mathrm{MeCN}$ & 0 & 26 & $\mathrm{PhCH}_{2} \mathrm{Cl}$ & $\mathrm{MeCN}$ & 0 \\
\hline 9 & $\mathrm{PhCOCl}$ & THF & 72 & 27 & & $\mathrm{MeCN}$ & 0 \\
\hline 10 & & $\mathrm{MeCN}$ & 70 & 28 & $\mathrm{Me}_{3} \mathrm{SiCl}$ & $\mathrm{MeCN}$ & 4 \\
\hline 11 & & THF & 68 & 29 & protic acid $^{\text {a }}$ & $\mathrm{MeCN}$ & $0-5$ \\
\hline 12 & & $\mathrm{MeCN}$ & 61 & 30 & $\mathrm{MeCOBr}$ & $\mathrm{MeCN}$ & 10 \\
\hline 13 & & $\mathrm{MeCN}$ & 0 & 31 & $\mathrm{BrCH}_{2} \mathrm{COBr}$ & $\mathrm{MeCN}$ & 28 \\
\hline 14 & $-\mathrm{COCl}$ & THF & 68 & 32 & $\mathrm{Ph}_{3} \mathrm{CCl}$ & THF & $37^{b}$ \\
\hline 15 & $\mathrm{CH}_{2}=\mathrm{CHCOCl}$ & $\mathrm{MeCN}$ & 70 & 33 & $\mathrm{Ph}_{3} \mathrm{CCl}$ & THF & $31^{\mathrm{b}, \mathrm{c}}$ \\
\hline 16 & trans $-\mathrm{MeCH}=\mathrm{CHCOCl}$ & $\mathrm{MeCN}$ & 69 & 34 & $\mathrm{Ph}_{3} \mathrm{CBr}$ & THF & 12 \\
\hline 17 & trans $-\mathrm{PhCH}=\mathrm{CHCOCl}$ & $\mathrm{MeCN}$ & 67 & 35 & $\left(p-\mathrm{Me}_{2} \mathrm{NC}_{6} \mathrm{H}_{4}\right)_{3} \mathrm{CCl}$ & THF & 0 \\
\hline 18 & $\mathrm{Me}_{2} \mathrm{C}=\mathrm{CHCOCl}$ & $\mathrm{MeCN}$ & 72 & & & & \\
\hline
\end{tabular}

(a) Introduced in the form of $4 \mathrm{~N} \mathrm{HCl}$ in dioxane, conc. $\mathrm{HCl}$, or acetic acid. (b) reaction time 48 h. (c) One equivalent of urea used instead of three. 
Previously reported chemistry of acylamidinium compounds and observed hydrolysis of formamidine ureas.

The known reactivity of acylamidinium compounds with nucleophiles is outlined in Scheme S3. Hydrolysis at the electrophilic iminium carbon atom has been observed to take two paths ( $a$ and $b$ ), while other nucleophiles can also attack the carbonyl carbon and thereby react according to paths $a-c .^{29-32}$ Our observed hydrolysis of $\mathbf{1 a}$ and $\mathbf{1 b}$ is shown in Equation 1.

Scheme S3. Reported routes of acylamidinium reactivity.

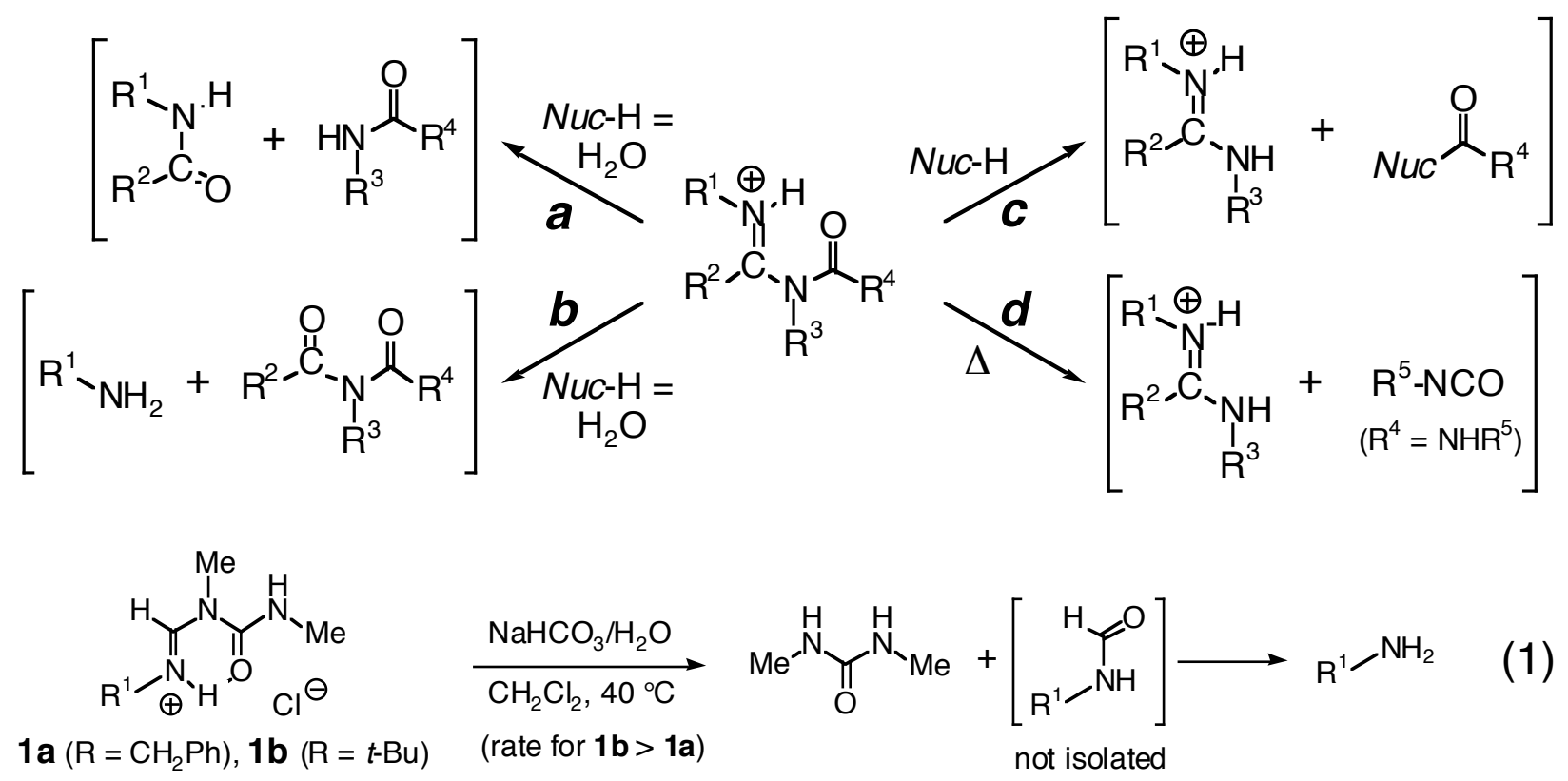

It should also be noted that allowing the urea-isocyanide condensation reaction to proceed too long causes a reduction in yield of formamidine urea. Thus, $\mathbf{1 b}$ was isolated (from 1 equiv. $t$-BuNC, 1.1 equiv. $\mathrm{MeCOCl}, 3$ equiv. 1,3-dimethylurea, THF; concentration $=0.6 \mathrm{M}$ in isonitrile) in the following yields as a function of reaction time: $1.5 \mathrm{~h}, 25 \% ; 3 \mathrm{~h}, 71 \% ; 6 \mathrm{~h}, 70 \%$; $9 \mathrm{~h}, 68 \% ; 12 \mathrm{~h}, 55 \% ; 16 \mathrm{~h}, 47 \% ; 24 \mathrm{~h}, 45 \% ; 36 \mathrm{~h}, 44 \% ; 48 \mathrm{~h}, 42 \%$. We do not yet know the fate of the presumably decomposed product at extended reaction times. 


\section{MATERIALS AND METHODS}

General. ${ }^{1} \mathrm{H}$ and ${ }^{13} \mathrm{C}$ NMR spectra were recorded on a Bruker DRX-500 and/or AMX-400 spectrometer in $\mathrm{CDCl}_{3}, \mathrm{CD}_{3} \mathrm{OD}, \mathrm{CD}_{3} \mathrm{CN}$ or DMSO- $d_{6}$ as solvent, which is indicated in each case. Mass spectra were taken using a HP 1100 LC/MS spectrometer (model G1946A) with mobile phase composed of 90:10 $\mathrm{CH}_{3} \mathrm{OH}: \mathrm{H}_{2} \mathrm{O}$ containing $0.1 \% \mathrm{CF}_{3} \mathrm{CO}_{2} \mathrm{H}$. Elemental analyses were performed by Midwest MicroLab, Inc. Melting points were measured in a Thomas Hoover capillary melting point apparatus and are uncorrected. Infrared spectra were recorded on MIDAC-FTIR or MAGNA-IR 550 spectrophotometers on solids dispersed on a $\mathrm{CaF}_{2}$ disc $(20 \mathrm{x}$ $2 \mathrm{~mm}$ ) or in $\mathrm{KBr}$ pellets. TLC analysis was facilitated by the use of the following stains in addition to UV light with fluorescent-indicating plates: phosphomolybdic acid, vanillin/EtOH, anisaldehyde/EtOH, or $\mathrm{KMnO}_{4} / \mathrm{H}_{2} \mathrm{O}$. THF, acetonitrile, diethyl ether, and toluene were dried by passage through activated alumina columns, ${ }^{33}$ dry DMF was purchased from Aldrich. Acid chlorides were purified by distillation immediately before use. Reactions requiring anhydrous conditions were performed under nitrogen.

\section{General procedure for formamidine-urea formation (Table 2).}

To a solution of isocyanide (1.6 mmol, 1.0 equiv) in dry $\mathrm{CH}_{3} \mathrm{CN}$ or THF $(2 \mathrm{~mL})$ at room temperature under a nitrogen atmosphere was added sequentially freshly distilled acyl chloride (1.76 mmol, 1.1 equiv) and the corresponding urea ( $4.8 \mathrm{mmol}, 3.0$ equiv). The reaction mixture was vigorously stirred for $9 \mathrm{~h}$, and the precipitate was filtered and washed carefully with a small amount of cold $\mathrm{CH}_{3} \mathrm{CN}$ or THF to remove colored impurities. The resulting products were white in color and analytically pure, except for the presence of small amounts of adsorbed water. The solids were dried in a vacuum oven at $40{ }^{\circ} \mathrm{C}$ and stored under nitrogen.

\section{Compound characterization.}

General. Examination of spectroscopic data for compounds 1 (Table 1) reveals the following characteristic resonances. ${ }^{1} \mathrm{H}$ NMR: ca. 2.9 and $3.4 \mathrm{ppm}$ (singlets, $3 \mathrm{H}$ each, urea NHMe), ca. 9.0 (broad s, 1H, C-H of formamidine group. ${ }^{13} \mathrm{C}$ NMR: ca. $153 \mathrm{ppm}(\mathrm{C}=\mathrm{O})$, ca. $150 \mathrm{ppm}(\mathrm{C}=\mathrm{N})$, ca. 33 and 26 ppm, (urea NHMe). IR: ca. $3300 \mathrm{~cm}^{-1}$ (N-H stretching vibration), ca. $1550 \mathrm{~cm}^{-1}$ ( $\mathrm{N}-\mathrm{H}$ bending vibration), $1650-1750 \mathrm{~cm}^{-1}(\mathrm{C}=\mathrm{O})$; note that the characteristic $2300-2800 \mathrm{~cm}^{-1}$ band of isonitriles are absent from the spectra of the formamidine urea products

1-(Benzylimino-methyl)-1,3-dimethyl-urea hydrochloride (1a). White solid (hygroscopic): Mp 157-158 ${ }^{\circ} \mathrm{C} ;{ }^{1} \mathrm{H}$ NMR (CD $\left.\mathrm{CD}_{3} \mathrm{OD}\right) \square 2.96$ (s, 3H), 3.41 (s, 3H), 4.91 (s, 2H), 7.47-7.54 (m, 5H), 9.19 (s, 1H); ${ }^{13} \mathrm{C}$ NMR $\left(\mathrm{CD}_{3} \mathrm{OD}\right) \square 25.6,29.6,50.9,126.9,127.2,127.5,133.5,151.4,154.8$; IR $\left(\mathrm{KBr}, \mathrm{cm}^{-1}\right)$ 3235, 2979, 1728, 1538, 699; MS m/z (relative intensity) $207(\mathrm{M}+2)^{+}(14), 206$ $(\mathrm{M}+1)^{+}(100), 108$ (12). HMRS calcd for $\mathrm{C}_{11} \mathrm{H}_{16} \mathrm{~N}_{3} \mathrm{O}(\mathrm{M}+1)^{+}$206.1293, found 206.1288.

1-(tert-Butylimino-methyl)-1,3-dimethyl-urea hydrochloride (1b). White solid (hygroscopic): Mp 180-181 ${ }^{\circ} \mathrm{C} ;{ }^{1} \mathrm{H}$ NMR (CD $\left.\mathrm{OD}\right) \square 1.57$ (s, 9H), 2.96 (s, 3H), 3.44 (s, 3H), 8.82 (br s, 1H); ${ }^{13} \mathrm{C}$ NMR $\left(\mathrm{CD}_{3} \mathrm{OD}\right) \square 26.8,27.3,31.5,58.1,152.5,153.5$; IR $\left(\mathrm{KBr}, \mathrm{cm}^{-1}\right) 3656,3216,3047,1654$; MS $m / z$ (relative intensity) $173(\mathrm{M}+2)^{+}(10), 172(\mathrm{M}+1)^{+}(100), 115$ (1). Anal. Calcd for $\mathrm{C}_{8} \mathrm{H}_{18} \mathrm{ClN}_{3} \mathrm{O} \cdot 1 / 4 \mathrm{H}_{2} \mathrm{O}: \mathrm{C}, 45.28 ; \mathrm{H}, 8.79 ; \mathrm{N}, 19.80$; Cl, 16.71. Found: C, 45.58; H, 8.71; N, 19.87; Cl, 16.66 . 
1-(Cyclohexylmethylimino-methyl)-1,3-dimethyl-urea hydrochloride (1c). White solid (hygroscopic): $\mathrm{Mp} 192-193{ }^{\circ} \mathrm{C} ;{ }^{1} \mathrm{H}$ NMR $\left(\mathrm{CD}_{3} \mathrm{OD}\right) \square 1.29-1.33$ (m, 1H), 1.47-1.50 (m, 2H), 1.58-1.63 (m, 2H), 1.78-1.80 (m, 1H), 1.94-1.97 (m, 2H), 2.08-2.11 (m, 2H), $2.95(\mathrm{~s}, 3 \mathrm{H}), 3.39$ $(\mathrm{s}, 3 \mathrm{H}), 3.75(\mathrm{~m}, 1 \mathrm{H}), 8.99$ (s, 1H); ${ }^{13} \mathrm{C}$ NMR $\left(\mathrm{CD}_{3} \mathrm{OD}\right) \square 24.3,24.9,26.1,27.2,30.8,32.8,60.1$, 150.9, 153.2; IR $\left(\mathrm{KBr}, \mathrm{cm}^{-1}\right) 3223,1720,1674,1532 ; \mathrm{MS} \mathrm{m} / z$ (relative intensity) $199(\mathrm{M}+2)^{+}$ (12), $200(\mathrm{M}+1)^{+}(100), 141$ (1). Anal. Calcd for $\mathrm{C}_{10} \mathrm{H}_{20} \mathrm{ClN}_{3} \mathrm{O}$ : C, 51.39; H, 8.62; N, 17.98; Cl, 15.17. Found: C, 51.34; H, 8.46; N, 17.98; Cl, 15.02.

[(1,3-Dimethyl-ureidomethylene)-amino]-acetic acid methyl ester hydrochloride (1d). White solid (hygroscopic): Mp 149-150 ${ }^{\circ} \mathrm{C} ;{ }^{1} \mathrm{H}$ NMR (DMSO-d $) ~ \square 2.80$ (s, 3H), 3.46 (s, 3H), 3.77 (s, $3 \mathrm{H}), 4.54$ (s, 2H), 9.84 (br s, 1H), 9.15 (br s, $1 \mathrm{H}$ ); ${ }^{13} \mathrm{C}$ NMR (DMSO- $d_{6}$ ) $\square 28.4,33.2,48.3,53.4$, 153.1, 158.3, 169.4; IR (KBr, cm ${ }^{-1}$ ) 3269, 2957, 1746, 1533; MS $\mathrm{m} / z$ (relative intensity) 189 $(\mathrm{M}+2)^{+}(9), 188(\mathrm{M}+1)^{+}(100), 119(4), 106$ (5). Anal. Calcd for $\mathrm{C}_{7} \mathrm{H}_{14} \mathrm{ClN}_{3} \mathrm{O}_{3} \cdot 1 / 2 \mathrm{H}_{2} \mathrm{O}: \mathrm{C}$, 36.14; H, 6.50; N, 18.06; Cl, 15.24. Found: C, 35.78; H, 6.21; N, 17.96; Cl, 15.62.

The ethyl ester was also prepared from the appropriate isocyanide (yield 69\%): [(1,3dimethyl-ureidomethylene)-amino]-acetic acid ethyl ester hydrochloride. White solid (hygroscopic): Mp 96-97 ${ }^{\circ} \mathrm{C} ;{ }^{1} \mathrm{H}$ NMR (DMSO- $d_{6}$ ) $\square 1.29$ (t, $J=9.1 \mathrm{~Hz}, 3 \mathrm{H}$ ), 2.81 (s, 3H), 3.44 $(\mathrm{s}, 3 \mathrm{H}), 4.22-4.26(\mathrm{~m}, 2 \mathrm{H}), 4.52(\mathrm{~s}, 2 \mathrm{H}), 8.58$ (br s, $1 \mathrm{H}), 9.04$ (br s, $1 \mathrm{H}) ;{ }^{13} \mathrm{C}$ NMR (DMSO- $\left.d_{6}\right) \square$ 14.9, 28.4, 33.1, 48.5, 62.3, 153.1, 158.3, 169.3; IR (KBr, $\left.\mathrm{cm}^{-1}\right) 3294,1738,1672,1527$; MS m/z (relative intensity) $203(\mathrm{M}+2)^{+}(10), 202(\mathrm{M}+1)^{+}(100), 119$ (2), 104 (31). Anal. Calcd for $\mathrm{C}_{8} \mathrm{H}_{16} \mathrm{ClN}_{3} \mathrm{O}_{3} \cdot \mathrm{H}_{2} \mathrm{O}: \mathrm{C}, 37.58 ; \mathrm{H}, 7.10 ; \mathrm{N}, 16.43 ; \mathrm{Cl}, 13.87$. Found: $\mathrm{C}, 37.26 ; \mathrm{H}, 6.83 ; \mathrm{N}, 15.99$; $\mathrm{Cl}, 14.22$.

1-Butyliminomethyl-1,3-dimethyl-urea hydrochloride (1e). White solid (hygroscopic): Mp 147-148 ${ }^{\circ} \mathrm{C} ;{ }^{1} \mathrm{H}$ NMR $\left(\mathrm{CD}_{3} \mathrm{OD}\right) \square 1.06(\mathrm{t}, J=7.3 \mathrm{~Hz}, 3 \mathrm{H}), 1.49(\mathrm{~m}, 2 \mathrm{H}), 1.79(\mathrm{~m}, 2 \mathrm{H}), 2.95$ (s, $3 \mathrm{H}), 3.41$ (s, 3H), 3.71 (br s, 2H), 9.01 (s, 1H); ${ }^{13} \mathrm{C}$ NMR $\left(\mathrm{CD}_{3} \mathrm{OD}\right) \square 13.0,19.6,27.2,31.3,31.9$, 49.9, 153.1, 155.9; IR (KBr, $\left.\mathrm{cm}^{-1}\right)$ 3217, 2963, 1714, 1678, 1528; MS m/z (relative intensity) 173 $(\mathrm{M}+2)^{+}(10), 172(\mathrm{M}+1)^{+}(100), 115$ (12). Anal. Calcd for $\mathrm{C}_{8} \mathrm{H}_{18} \mathrm{ClN}_{3} \mathrm{O} \bullet 1 / 4 \mathrm{H}_{2} \mathrm{O}$ : C, 45.28; $\mathrm{H}$, 8.79; N, 19.80; Cl, 16.71. Found: C, 45.36; H, 8.62; N, 19.99; Cl, 17.89.

1-(Benzylimino-methyl)-1,3-dimethyl-thiourea hydrochloride (1f). White solid (hygroscopic): Mp 160-161 ${ }^{\circ} \mathrm{C} ;{ }^{1} \mathrm{H}$ NMR $\left(\mathrm{CD}_{3} \mathrm{OD}\right) \square 3.37$ (s, 3H), 3.60 (s, 3H), 4.92 (s, 2H), 7.47-7.54 (m, 5H), 9.49 (br s, $1 \mathrm{H}) ;{ }^{13} \mathrm{C}$ NMR $\left(\mathrm{CD}_{3} \mathrm{OD}\right) \square 31.9,36.1,52.4,128.5,128.9,129.1,135.0,157.1,182.6$; IR $\left(\mathrm{KBr}, \mathrm{cm}^{-1}\right)$ 3156, 2974, 1669, 1549, 699; MS m/z (relative intensity) $223(\mathrm{M}+2)^{+}(14), 222$ $(\mathrm{M}+1)^{+}(100), 175$ (6), 144 (1). Anal. Calcd for $\mathrm{C}_{11} \mathrm{H}_{16} \mathrm{ClN}_{3} \mathrm{~S} \bullet 1 / 2 \mathrm{H}_{2} \mathrm{O}: \mathrm{C}, 49.52 ; \mathrm{H}, 6.42 ; \mathrm{N}$, 15.75; S, 12.02; Cl, 13.29. Found: C, 49.78; H, 6.43; N, 16.19; S, 12.26; Cl, 13.39.

1-(Benzylimino-methyl)-imidazolidin-2-one hydrochloride (1g). White solid (hygroscopic): Mp 182-183 ${ }^{\circ} \mathrm{C} ;{ }^{1} \mathrm{H}$ NMR $\left(\mathrm{CD}_{3} \mathrm{OD}\right) \square 3.66(\mathrm{t}, J=7.0 \mathrm{~Hz}, 2 \mathrm{H}), 3.88(\mathrm{t}, J=7.0 \mathrm{~Hz}, 2 \mathrm{H}), 4.21(\mathrm{~s}, 2 \mathrm{H})$, 7.49-7.58 (m, 5H), 8.89 (br s, $1 \mathrm{H}) ;{ }^{13} \mathrm{C}$ NMR (CD $\left.\mathrm{OD}\right) \square 37.6,39.3,40.9,43.4,128.6,128.9$, 129.2, 133.5, 160.8, 166.6; IR $\left(\mathrm{KBr}, \mathrm{cm}^{-1}\right) 3221,2971,1770,1679,700 ; \mathrm{MS} \mathrm{m} / \mathrm{z}$ (relative intensity) $205(\mathrm{M}+2)^{+}(14), 204(\mathrm{M}+1)^{+}(100), 119(5), 105$ (1). Anal. Calcd for $\mathrm{C}_{11} \mathrm{H}_{14} \mathrm{ClN}_{3} \mathrm{O}$ 1/4 $\mathrm{H}_{2} \mathrm{O}$ : C, 54.10; H, 5.98; N, 17.21; Cl, 14.52. Found: C, 54.05; H, 5.98; N, 17.09; Cl, 14.02.

1-(Benzylimino-methyl)-1-methyl-urea hydrochloride (1h). White solid (hygroscopic): Mp 168-169 ${ }^{\circ} \mathrm{C} ;{ }^{1} \mathrm{H}$ NMR $\left(\mathrm{CD}_{3} \mathrm{OD}\right) \square 3.41(\mathrm{~s}, 3 \mathrm{H}), 4.91(\mathrm{~s}, 2 \mathrm{H}), 7.50-7.52(\mathrm{~m}, 5 \mathrm{H}), 9.20$ (br s, $\left.1 \mathrm{H}\right)$; ${ }^{13} \mathrm{C} \mathrm{NMR}\left(\mathrm{CD}_{3} \mathrm{OD}\right) \square 30.1,56.3,131.5,132.2,132.6,132.9,137.4,156.3,162.2 ; \mathrm{IR}\left(\mathrm{KBr}, \mathrm{cm}^{-1}\right)$ 3274, 2829, 1732, 1687, 1359, 701; MS m/z (relative intensity) $193(\mathrm{M}+2)^{+}(12), 192(\mathrm{M}+1)^{+}$ 
(100), 149 (2), 119 (4), 101 (4). Anal. Calcd for $\mathrm{C}_{10} \mathrm{H}_{14} \mathrm{ClN}_{3} \mathrm{O} \cdot 1 / 4 \mathrm{H}_{2} \mathrm{O}: \mathrm{C}, 51.73 ; \mathrm{H}, 6.29$; N, 18.10; Cl, 15.27. Found: C, 51.95; H, 6.15; N, 18.57; Cl, 14.97.

1-Adamantan-1-yl-1-(benzylimino-methyl)-urea hydrochloride (1i). White solid (hygroscopic): Mp 171-172 ${ }^{\circ} \mathrm{C} ;{ }^{1} \mathrm{H}$ NMR $\left(\mathrm{CDCl}_{3}\right) \square 1.65$ (br s, 6H), 1.66-2.06 (m, 9H), 4.48 (s, 2H), 5.31 (br s, $1 \mathrm{H}), 7.25-7.34(\mathrm{~m}, 5 \mathrm{H}), 8.53(\mathrm{~s}, 1 \mathrm{H}) ;{ }^{13} \mathrm{C} \mathrm{NMR}\left(\mathrm{CDCl}_{3}\right) \square 30.8,37.2,42.3,53.3,129.2,129.9$, 130.3, 134.5, 149.4, 153.2; IR (KBr, $\left.\mathrm{cm}^{-1}\right)$ 3240, 2918, 1747, 1686, 1555, 700; MS m/z (relative intensity) $334(\mathrm{M}+\mathrm{Na})^{+}(11), 313(\mathrm{M}+2)^{+}(22), 312(\mathrm{M}+1)^{+}(100), 152(18), 119(8), 101$ (8). Anal. Calcd for $\mathrm{C}_{19} \mathrm{H}_{26} \mathrm{ClN}_{3} \mathrm{O} \bullet 1 / 2 \mathrm{H}_{2} \mathrm{O}: \mathrm{C}$, 63.94; H, 7.63; N, 11.77; Cl, 9.93. Found: C, 63.88; $\mathrm{H}, 7.46 ; \mathrm{N}, 11.63 ; \mathrm{Cl}, 10.18$.

1-(Benzylimino-methyl)-1-phenyl-urea hydrochloride (1j). Yellow solid (hygroscopic): Mp 176-177 ${ }^{\circ} \mathrm{C}$; ${ }^{1} \mathrm{H}$ NMR (DMSO-d $)$ $\square 7.10-7.58$ (m, 10H), 9.12 (s, 1H), 10.92 (br s, 2H); ${ }^{13} \mathrm{C}$ NMR (DMSO- $\left.d_{6}\right) \square 48.0,118.5,119.7,122.3,127.9,129.6,130.1,135.1,138.2,140.2$, 155.2; IR $\left(\mathrm{KBr}, \mathrm{cm}^{-1}\right) 3266,2890,1750,1687,1561,1301,700$; MS m/z (relative intensity) $276(\mathrm{M}+\mathrm{Na})^{+}$ (14), $255(\mathrm{M}+2)^{+}(17), 254(\mathrm{M}+1)^{+}(100), 225(15), 119$ (5). Anal. Calcd for $\mathrm{C}_{15} \mathrm{H}_{16} \mathrm{ClN}_{3} \mathrm{O}: \mathrm{C}$, 62.18; H, 5.57; N, 14.50; Cl, 12.24. Found: C, 62.40; H, 5.57; N, 14.77; Cl, 12.39.

1-Benzyl-1-(benzylimino-methyl)-urea hydrochloride (1k). White solid (hygroscopic): Mp 177-178 ${ }^{\circ} \mathrm{C} ;{ }^{1} \mathrm{H}$ NMR (DMSO- $\left.d_{6}\right) \square 4.49$ (s, 2H), 4.84 (s, 2H), 8.53 (br s, 2H), 7.33-7.54 (m, $10 \mathrm{H}), 9.03$ (s, 1H); ${ }^{13} \mathrm{C}$ NMR (DMSO-d $) \square 44.0,48.0,128.1,129.6,135.1,139.0,152.9,158.0$; IR $\left(\mathrm{KBr}, \mathrm{cm}^{-1}\right) 3270,2879,1751,1685,1548,1301,699 ;$ MS $\mathrm{m} / \mathrm{z}$ (relative intensity) 290 $(\mathrm{M}+\mathrm{Na})^{+}(8), 269(\mathrm{M}+2)^{+}(20), 268(\mathrm{M}+1)^{+}(100), 119$ (2). Anal. Calcd for $\mathrm{C}_{16} \mathrm{H}_{18} \mathrm{ClN}_{3} \mathrm{O}: \mathrm{C}$, 63.26; H, 5.97; N, 13.83; Cl, 11.67. Found: C, 62.90; H, 5.91; N, 13.90; Cl, 11.42.

1,3-Dimethyl-1-[(toluene-4-sulfonylmethylimino)-methyl]-urea hydrochloride (11). White solid (hygroscopic): $\mathrm{Mp} 114-115{ }^{\circ} \mathrm{C} ;{ }^{1} \mathrm{H}$ NMR $\left(\mathrm{CD}_{3} \mathrm{OD}\right) \square$ 1.28-1.32 (m, 1H), 1.48 (ddd, $\mathrm{J}=13.2$, 13.2, 9.6 Hz, 2H), 1.60 (ddd, J=13.2, 13.2, $9.6 \mathrm{~Hz}, 2 \mathrm{H}), 1.78-1.80(\mathrm{~m}, 1 \mathrm{H}), 1.95$ (d, J= 11.6 Hz, 2H), 2.09 (d, J= 11.7 Hz, 2H), $2.95(\mathrm{~s}, 3 \mathrm{H}), 3.38(\mathrm{~s}, 3 \mathrm{H}), 3.71-3.76(\mathrm{~m}, 1 \mathrm{H}), 9.0(\mathrm{~s}, 1 \mathrm{H}) ;{ }^{13} \mathrm{C} \mathrm{NMR}$ $\left(\mathrm{CD}_{3} \mathrm{OD}\right) \square 26.3,26.7,35.1,74.4,124.7,125.9,128.9,130.0,135.4,158.8,162.3$; IR $\left(\mathrm{KBr}, \mathrm{cm}^{-1}\right)$ 3320, 1715, 1530, 1349, 703; MS m/z (relative intensity) $306(\mathrm{M}+\mathrm{Na})^{+}(63), 285(\mathrm{M}+2)^{+}(17)$, $284(\mathrm{M}+1)^{+}(100), 177$ (23), 157 (18), 105 (5). Anal. Calcd for $\mathrm{C}_{12} \mathrm{H}_{18} \mathrm{ClN}_{3} \mathrm{O}_{3} \mathrm{~S} \bullet 1 / 2 \mathrm{H}_{2} \mathrm{O}: \mathrm{C}$, 43.83; H, 5.82; N, 12.78; Cl, 10.78; S, 9.75. Found: C, 43.99; H, 6.09; N, 13.15; Cl, 10.45; S, 9.62 .

1-(\{6-[(1,3-Dimethyl-ureidomethylene)-amino]-hexylimino\}-methyl)-1,3-dimethyl-urea bishydrochloride (1m). Beige solid (hygroscopic): Mp $153-154{ }^{\circ} \mathrm{C} ;{ }^{1} \mathrm{H}$ NMR $\left(\mathrm{CD}_{3} \mathrm{OD}\right) \square 1.54$ (m, $4 \mathrm{H}), 1.85(\mathrm{~m}, 4 \mathrm{H}), 2.95(\mathrm{~s}, 6 \mathrm{H}), 3.39(\mathrm{~s}, 3 \mathrm{H}), 3.74(\mathrm{br} \mathrm{s}, 4 \mathrm{H}), 9.02(\mathrm{~s}, 2 \mathrm{H}) ;{ }^{13} \mathrm{C} \mathrm{NMR}\left(\mathrm{CD}_{3} \mathrm{OD}\right) \square$ 25.7, 26.4, 27.2, 29.5, 31.8, 49.8, 153.1, 156.0; IR (KBr, $\left.\mathrm{cm}^{-1}\right) 3213,2940,1721,1672,1533$; MS $m / z$ (relative intensity) $335(\mathrm{M}+\mathrm{Na})^{+}(35), 313(\mathrm{M}+1)^{+}(63), 243(31), 157$ (100). Anal. Calcd for $\mathrm{C}_{14} \mathrm{H}_{30} \mathrm{Cl}_{2} \mathrm{~N}_{6} \mathrm{O}_{2} \bullet 1 / 2 \mathrm{H}_{2} \mathrm{O}: \mathrm{C}, 42.64 ; \mathrm{H}, 7.92 ; \mathrm{N}, 21.31 ; \mathrm{Cl}, 17.98$. Found: $\mathrm{C}, 42.35, \mathrm{H}$, 7.80; N, 21.53; Cl, 17.44 .

1-[(2,6-Dimethyl-phenylimino)-methyl]-3-methyl-urea hydrochloride (1n). White solid (hygroscopic): $\mathrm{Mp} 167-168{ }^{\circ} \mathrm{C} ;{ }^{1} \mathrm{H}$ NMR $\left(\mathrm{CD}_{3} \mathrm{OD}\right) \square 2.50$ (s, 3H), 2.80 (s, 3H), 2.98 (s, 3H), 3.39 (s, 3H), 7.27-8.16 (m, 5H), 8.17 (s, 1H); ${ }^{13} \mathrm{C}$ NMR $\left(\mathrm{CD}_{3} \mathrm{OD}\right) \square 17.2,17.5,26.1,129.6$, 132.0, 134.8; IR (KBr, cm $\left.{ }^{-1}\right) 3310,2869,1654,720,697$; MS m/z (relative intensity) $221(\mathrm{M}+2)^{+}$ 
(14), $220(\mathrm{M}+1)^{+}(100), 119$ (2). Anal. Calcd for $\mathrm{C}_{12} \mathrm{H}_{18} \mathrm{ClN}_{3} \mathrm{O} \bullet 1 / 4 \mathrm{H}_{2} \mathrm{O}: \mathrm{C}, 55.38 ; \mathrm{H}, 7.17$; , $16.15 ; \mathrm{Cl}, 13.62$. Found: C, 55.54, H, 7.09; N, 13.74; Cl, 16.10.

1,3-Diallyl-1-(benzylimino-methyl)-urea hydrochloride (1o). Colorless oil: ${ }^{1} \mathrm{H}$ NMR $\left(\mathrm{CD}_{3} \mathrm{CN}\right) \square$ $3.93(\mathrm{~d}, J=15.8 \mathrm{~Hz}, 2 \mathrm{H}), 4.45(\mathrm{~s}, 1 \mathrm{H}), 4.83$ (br s, 1H), 5.16-5.29 (m, 4H), 5.93-5.99 (m, 2H), 7.41-7.55 (m, 5H), 9.30 (br d, 1H); ${ }^{13} \mathrm{C}$ NMR (CD $\left.3 \mathrm{OD}\right) \square 42.3,45.9,51.0,114.3,127.9,129.1$, 132.5, 136.0, 155.3; IR $\left(\mathrm{CaF}_{2}, \mathrm{~cm}^{-1}\right)$ 3305, 2986, 1703, 1562, 1241, 921; MS $\mathrm{m} / z$ (relative intensity) $259(\mathrm{M}+2)^{+}(20), 258(\mathrm{M}+1)^{+}(100), 183(13), 141(15)$. Anal. Calcd for $\mathrm{C}_{15} \mathrm{H}_{20} \mathrm{ClN}_{3} \mathrm{O}$ : C, 61.32; H, 6.86; N, 14.30; Cl, 12.07. Found: C, 61.02, H, 7.01; N, 14.45; Cl, 11.75.

1-Allyl-1-(benzylimino-methyl)-urea hydrochloride (1p). Colorless oil: ${ }^{1} \mathrm{H}$ NMR $\left(\mathrm{CDCl}_{3}\right) \square 3.91$ $(\mathrm{d}, J=15.9 \mathrm{~Hz}, 2 \mathrm{H}), 4.49$ (s, 1H), $4.61(\mathrm{~s}, 1 \mathrm{H}), 5.16-5.30(\mathrm{~m}, 2 \mathrm{H}), 5.89-5.94(\mathrm{~m}, 1 \mathrm{H}), 7.36-7.41$ $(\mathrm{m}, 5 \mathrm{H}), 8.67(\mathrm{~s}, 1 \mathrm{H}), 9.31(\mathrm{~s}, 1 \mathrm{H}) ;{ }^{13} \mathrm{C} \mathrm{NMR}\left(\mathrm{CDCl}_{3}\right) \square 43.3,45.8,116.2,127.8,128.3,129.2$, 135.2, 137.5, 158.1; IR $\left(\mathrm{CaF}_{2}, \mathrm{~cm}^{-1}\right) 3307,2950,1715,1563,1245 ; \mathrm{MS} m / z$ (relative intensity) $240(\mathrm{M}+\mathrm{Na})^{+}(16), 219(\mathrm{M}+2)^{+}(16), 218(\mathrm{M}+1)^{+}(100), 150(7), 136$ (13), 101 (60). Anal. Calcd for $\mathrm{C}_{12} \mathrm{H}_{16} \mathrm{ClN}_{3} \mathrm{O}: \mathrm{C}, 56.80 ; \mathrm{H}, 6.36 ; \mathrm{N}, 16.56 ; \mathrm{Cl}, 13.97$. Found: C, 57.12, H, 6.48; N, 16.77; Cl, 13.58 .

4-Methyl-benzoic acid N'-(benzylimino-methyl)-hydrazide hydrochloride (3). White solid (hygroscopic): $\mathrm{Mp} 169-170{ }^{\circ} \mathrm{C} ;{ }^{1} \mathrm{H} \mathrm{NMR}\left(\mathrm{CDCl}_{3}\right) \square 2.13$ (s, 1H), 2.48 (s, 3H), 3.38 (s, 1H), 4.74 (s, 2H), 7.36-7.53 (m, 9H), 7.85-7.91 (m, 3H); ${ }^{13} \mathrm{C} \mathrm{NMR}\left(\mathrm{CDCl}_{3}\right) \square 21.6,50.3,128.1,128.7$, 129.4, 143.2, 155.7; IR (KBr, cm ${ }^{-1}$ ) 3062, 2924, 1707, 1652, 1495, 1269, 700; MS m/z (relative intensity) $290(\mathrm{M}+\mathrm{Na})^{+}(14), 269(\mathrm{M}+2)^{+}(18), 268(\mathrm{M}+1)^{+}(100), 119(5)$. Anal. Calcd for $\mathrm{C}_{16} \mathrm{H}_{18} \mathrm{ClN}_{3} \mathrm{O}: \mathrm{C}, 63.26 ; \mathrm{H}, 5.97 ; \mathrm{N}, 13.83 ; \mathrm{Cl}, 11.67$. Found: C, 62.93; H, 5.99; N, 13.80; Cl, 11.30 .

Acylurea Byproducts. In all cases in which the soluble portions of reaction mixtures were examined, the appropriate acylurea was detected in approximately equimolar amounts relative to the formamidine ureas formed. To support the assignment of acylurea structure, the examples were characterized as described below.
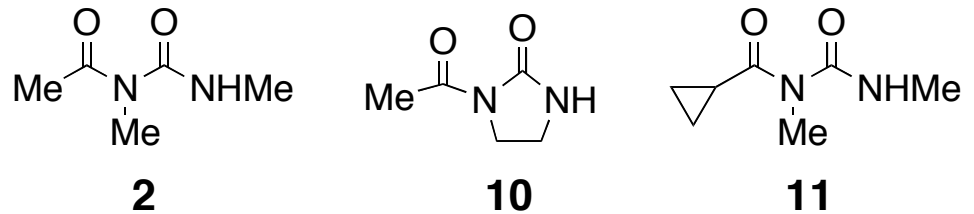

11

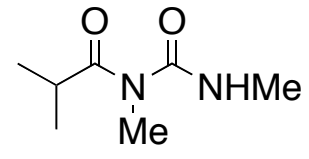

12

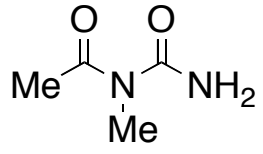

13

Compounds $\mathbf{2}^{34-36}$ and $\mathbf{1 0}^{37}$ were identified from comparison to literature values.

1-Cyclopropanecarbonyl-1,3-dimethyl-urea (11). Colorless oil: ${ }^{1} \mathrm{H}$ NMR ( $\left.\mathrm{CD}_{3} \mathrm{OD}\right) \square 1.03-1.07$ (m, 4H), 2.18-2.20 (m, 1H), 2.89 (s, 3H), 3.48 (s, 3H); ${ }^{13} \mathrm{C}$ NMR (CD 3 OD) $\square 9.0,14.1,26.3$, 31.4, 156.7, 177.8; IR $\left(\mathrm{CaF}_{2}, \mathrm{~cm}^{-1}\right)$ 3296, 2951, 1720, 1650; MS $\mathrm{m} / z$ (relative intensity) 179 $(\mathrm{M}+\mathrm{Na})^{+}(16), 158(\mathrm{M}+2)^{+}(9), 158(\mathrm{M}+1)^{+}(100), 105(2)$. Anal. Calcd for $\mathrm{C}_{7} \mathrm{H}_{12} \mathrm{~N}_{2} \mathrm{O}_{2}$ : C, 53.83; H, 7.74; N, 17.94. Found: C, 53.68, H, 7.70; N, 17.71.

1-Isobutyryl-1,3-dimethyl-urea (12). Colorless oil: ${ }^{1} \mathrm{H}$ NMR $\left(\mathrm{CD}_{3} \mathrm{OD}\right) \square 1.21$ (d, $J=2.2 \mathrm{~Hz}$, $6 \mathrm{H}), 2.89$ (s, 3H), 3.17-3.20 (m, 1H), 3.36 (s, 3H); ${ }^{13} \mathrm{C} \mathrm{NMR}\left(\mathrm{CD}_{3} \mathrm{OD}\right) \square 18.7,26.4,31.2,33.8$, 157.1, 181.4; IR $\left(\mathrm{CaF}_{2}, \mathrm{~cm}^{-1}\right) 3297,2974,1720,1658 ; \mathrm{MS} \mathrm{m} / z$ (relative intensity) $181(\mathrm{M}+\mathrm{Na})^{+}$ 
(17), $160(\mathrm{M}+2)^{+}(9), 159(\mathrm{M}+1)^{+}(100), 105$ (4). Anal. Calcd for $\mathrm{C}_{7} \mathrm{H}_{14} \mathrm{~N}_{2} \mathrm{O}_{2}$ : C, 53.15; H, 8.92; N, 17.71. Found: C, 53.21, H, 8.89; N, 17.72.

1-Acetyl-1-methyl-urea (13). White solid: Mp 159-160 ${ }^{\circ} \mathrm{C} ;{ }^{1} \mathrm{H}$ NMR $\left(\mathrm{CD}_{3} \mathrm{OD}\right) \square 2.16$ (s, 3H), 2.89 (s, 3H); ${ }^{13} \mathrm{C}$ NMR (CD $\left.\mathrm{OD}\right) \square 22.6,25.2,155.4,173.1$; IR $\left(\mathrm{KBr}, \mathrm{cm}^{-1}\right) 3221,1715,1559$; MS $m / z$ (relative intensity) $139(\mathrm{M}+\mathrm{Na})^{+}(91), 118(\mathrm{M}+2)^{+}(5), 117(\mathrm{M}+1)^{+}(100)$. Anal. Calcd for $\mathrm{C}_{4} \mathrm{H}_{8} \mathrm{~N}_{2} \mathrm{O}_{2}$ : C, 41.37; H, 6.94; N, 24.12. Found: C, 41.52, H, 6.82; N, 23.82.

\section{References and Notes}

(1) Marmet, D.; Boullanger, P.; Descotes, G. Tetrahedron Lett. 1980, 21, 1459-1462.

(2) Hegarty, A. F.; Chandler, A. Tetrahedron Lett. 1980, 21, 885-888.

(3) Gottlieb, L.; Meyers, A. I. Tetrahedron Lett. 1990, 31, 4723-4726.

(4) Hashida, Y.; Imai, A.; Sekiguchi, S. J. Heterocycl. Chem. 1989, 26, 901-905.

(5) Meyers, A. I.; Bailey, T. R. Journal of Organic Chemistry 1986, 51, 872-875.

(6) Loewe, M. F.; Meyers, A. I. Tetrahedron Letters 1985, 26, 3291-3294.

(7) Smith, R.; Livinghouse, T. Synthetic Communications 1984, 14, 639-646.

(8) Ito, Y.; Inubushi, Y.; Saegusa, T. Tetrahedron Letters 1974, 1283-1286.

(9) Saegusa, T.; Ito, Y.; Kobayashi, S.; Hirota, K.; Yoshioka, H. Bulletin of the Chemical Society of Japan 1969, 42, 3310-3313.

(10) Saegusa, T.; Ito, Y.; Kobayashi, S.; Hirota, K.; Yoshioka, H. Tetrahedron Letters 1966, 6121-6124.

(11) Bertani, R.; Crociani, L.; D'Arcangelo, G.; Rossetto, G.; Traldi, P.; Zanella, P. J. Organomet. Chem. 2001, 626, 11-15.

(12) Jakobsen, P.; Treppendahl, S. Tetrahedron 1977, 33, 3137-3140.

(13) Jakobsen, P. Acta Chemica Scandinavica, Series B: Organic Chemistry and Biochemistry 1976, B30, 847-852.

(14) Mitin, Y. V.; Glushenkova, V. R.; Vlasov, G. P. Zh. Obshch. Khim. 1962, 32, 3867-3871.

(15) Saluste, C. G.; Whitby, R. J.; Furber, M. Tetrahedron Lett. 2001, 42, 6191-6194.

(16) Katritzky, A. R.; Button, M. A. C.; Busont, S. J. Org. Chem. 2001, 66, 2865-2868.

(17) Simon, J. R. Synthesis 2001, 2011-2014.

(18) Bergemann, M.; Neidlein, R. Helv. Chim. Acta 1999, 82, 909-918.

(19) Bossio, R.; Marcaccini, S.; Pepino, R. J. Heterocycl. Chem. 1995, 32, 1115-1116.

(20) Bossio, R.; Marcaccini, S.; Pepino, R. Tetrahedron Lett. 1995, 36, 2325-2326.

(21) Morel, G.; Marchand, E.; Foucaud, A.; Toupet, L. Journal of Organic Chemistry 1989, 54, $1185-1191$.

(22) Witczak, Z. J. Tetrahedron 1985, 41, 4781-4785.

(23) Ugi, I. Isonitrile Chemistry; Academic Press: New York, 1971.

(24) Westling, M.; Smith, R.; Livinghouse, T. J. Org. Chem. 1986, 51, 1159-1165.

(25) Westling, M.; Livinghouse, T. Tetrahedron Lett. 1985, 26, 5389-5392.

(26) Westling, M.; Livinghouse, T. J. Am. Chem. Soc. 1987, 109, 590-592.

(27) Lee, C. H.; Westling, M.; Livinghouse, T.; Williams, A. C. J. Am. Chem. Soc. 1992, 114, 4089-4095.

(28) Newton, C. G.; Ollis, W. D.; Wright, D. E. jcspt1 1984, 1, 75-84.

(29) Glocker, M. O.; Shrestha-Davadi, P. B.; Küchler-Krischun, J.; Hofmann, J.; Fischer, H.; Jochims, J. C. Chem. Ber. 1993, 126, 1859-1865. 
(30) Jochims, J. C.; Glocker, M. O. Chem. Ber. 1990, 123, 1537-1544.

(31) Bitter, I.; Kárpáti-Ádám, E.; Tóke, L. Acta Chim. Acad. Sci. Hung. 1979, 102, 235-246.

(32) Csuros, Z.; Soos, R.; Bitter, I.; Karpati-Adam, E. Acta Chim. (Budapest) 1972, 73, 239246.

(33) Pangborn, A. B.; Giardello, M. A.; Grubbs, R. H.; Rosen, R. K.; Timmers, F. J. Organometallics 1996, 15, 1518-1520.

(34) Egg, H.; Volgger, I. Synthesis 1982, 12, 1071-1073.

(35) Dmitrichenko, M. Y.; Rozinov, V. G.; Donskikh, V. I.; Ratovskii, G. V.; Sergienko, L. M.; Dolgushin, G. V.; Valeev, R. B. Zh. Obsch. Khim. 1988, 58, 2252-2261.

(36) Hamed, A.; Ismail, A.; Hitzler, M. G.; Jochims, J. C. J. Prakt. Chem./Chem.-Ztg. 1995, 337, 395-390.

(37) Greenhalgh, R.; Weinberger, M. A. Can. J. Chem. 1965, 43, 3340-3346.

\section{X-RAY CRYSTALLOGRAPHY}

Crystals of 1a were obtained from acetonitrile solution.

DETAILS OF CRYSTALLOGRAPHY APPEAR IN A CIF FILE, AVAILABLE SEPARATELY FROM THE AUTHORS (mgfinn@scripps.edu). 